\title{
Positive Solution for the Nonlinear Hadamard Type Fractional Differential Equation with $p$-Laplacian
}

\author{
Ya-ling Li and Shi-you Lin \\ School of Mathematics and Statistics, Hainan Normal University, Haikou, Hainan 571158, China \\ Correspondence should be addressed to Shi-you Lin; linsy1111@aliyun.com
}

Received 28 May 2013; Revised 6 August 2013; Accepted 7 August 2013

Academic Editor: T. Diagana

Copyright (c) 2013 Y.-1. Li and S.-y. Lin. This is an open access article distributed under the Creative Commons Attribution License, which permits unrestricted use, distribution, and reproduction in any medium, provided the original work is properly cited.

We study the following nonlinear fractional differential equation involving the $p$-Laplacian operator $D^{\beta}\left(\varphi_{p}\left(D^{\alpha} u(t)\right)\right)=f(t, u(t))$, $1<t<e, u(1)=u^{\prime}(1)=u^{\prime}(e)=0, D^{\alpha} u(1)=D^{\alpha} u(e)=0$, where the continuous function $f:[1, e] \times[0,+\infty) \rightarrow[0,+\infty), 2<$ $\alpha \leq 3,1<\beta \leq 2$. $D^{\alpha}$ denotes the standard Hadamard fractional derivative of the order $\alpha$, the constant $p>1$, and the $p$-Laplacian operator $\varphi_{p}(s)=|s|^{p-2} s$. We show some results about the existence and the uniqueness of the positive solution by using fixed point theorems and the properties of Green's function and the $p$-Laplacian operator.

\section{Introduction}

Fractional differential equations have attracted more and more attention for their useful applications in various fields, such as economics, science, and engineering; see [1-4]. In the last few decades, much attention has been focused on the study of the existence and uniqueness of solutions for boundary value problems of Riemann-Liouville type or Caputo type fractional differential equations; see [5-19]. There are few papers devoted to the research of the $p$-Laplacian fractional differential equations; see [20-25].

By the use of the fixed point theorem on cones, Chai in [20] obtained the existence and multiplicity of positive solutions for a class of boundary value problem of fractional differential equation with $p$-Laplacian operator:

$$
\begin{aligned}
& D_{0+}^{\beta}\left(\phi_{p}\left(D_{0+}^{\alpha} u(t)\right)\right)=f(t, u(t)), \quad 0<t<1, \\
& u(0)=0, \quad u(1)+\sigma D_{0+}^{\gamma} u(1)=0, \quad D_{0+}^{\alpha} u(0)=0,
\end{aligned}
$$

where $1<\alpha \leq 2,0<\beta \leq 1,0<\gamma \leq 1,0 \leq \alpha-\gamma-1, \sigma$ is a positive constant number, and $D_{0+}^{\alpha}, D_{0+}^{\beta}, D_{0+}^{\gamma}$ are the standard Riemann-Liouville derivatives. $\phi_{p}(s)=|s|^{p-2} s, p>1, \phi_{p}^{-1}=$ $\phi_{q}, 1 / p+1 / q=1$.
Han et al. in [22] studied the following boundary value problem of nonlinear fractional differential equation with $p$-Laplacian operator:

$$
\begin{aligned}
D_{0+}^{\beta}\left(\phi_{p}\left(D_{0+}^{\alpha} u(t)\right)\right) & +a(t) f(u)=0, \quad 0<t<1, \\
u(0) & =\gamma u(\xi)+\lambda, \\
\phi_{p}\left(D_{0+}^{\alpha} u(0)\right) & =\left(\phi_{p}\left(D_{0+}^{\alpha} u(1)\right)\right)^{\prime} \\
& =\left(\phi_{p}\left(D_{0+}^{\alpha} u(1)\right)\right)^{\prime \prime}=0,
\end{aligned}
$$

where $0<\alpha \leq 1,2<\beta \leq 3,0 \leq \gamma<1,0 \leq \xi \leq 1$, $\lambda>0$ is a parameter, and $D_{0+}^{\alpha}, D_{0+}^{\beta}$ are the standard Caputo fractional derivatives. By the properties of Green function and Schauder fixed point theorem, several existence and nonexistence results and the uniqueness of positive solutions are acquired.

Liu et al. in [23] investigated the solvability of a fractional differential equation model involving the $p$-Laplacian operator with boundary value conditions as follows:

$$
\left(\varphi_{p}\left({ }^{c} D^{\alpha} x(t)\right)\right)^{\prime}=f(t, x(t)), \quad t \in(0,1),
$$




$$
\begin{gathered}
x(0)=r_{0} x(1), \quad x^{\prime}(0)=r_{1} x^{\prime}(1), \\
x^{(i)}=0, \quad i=2,3, \ldots,[\alpha]-1,
\end{gathered}
$$

where $1<\alpha \in \mathbb{R}, r_{0}, r_{1} \neq 1$, and ${ }^{c} D^{\alpha}$ is the standard Caputo derivative. By the means of the Banach contraction mapping principle, they obtained the existence and uniqueness of a solution for the model.

$\mathrm{Lu}$ et al. in [24] considered the following fractional boundary value problem with $p$-Laplacian operator:

$$
\begin{gathered}
D_{0+}^{\beta}\left(\phi_{p}\left(D_{0+}^{\alpha} u(t)\right)\right)=f(t, u(t)), \quad 0<t<1, \\
u(0)=u^{\prime}(0)=u^{\prime}(1)=0, \quad D_{0+}^{\alpha} u(0)=D_{0+}^{\beta} u(1)=0,
\end{gathered}
$$

where $2<\alpha \leq 3,1<\beta \leq 2$, and $D_{0+}^{\alpha}, D_{0+}^{\beta}$ are the standard Riemann-Liouville fractional derivatives. By the properties of Green's function, the Guo-Krasnosel'skii fixed point theorem, the Leggett-Williams fixed point theorem, and the upper and lower solutions method, some new results on the existence of positive solutions are gained.

Motivated by the mentioned papers, we will consider the Hadamard fractional boundary value with $p$-Laplacian operator as below:

$$
\begin{gathered}
D^{\beta}\left(\varphi_{p}\left(D^{\alpha} u(t)\right)\right)=f(t, u(t)), \quad 1<t<e, \\
u(1)=u^{\prime}(1)=u^{\prime}(e)=0, \quad D^{\alpha} u(1)=D^{\alpha} u(e)=0,
\end{gathered}
$$

where $2<\alpha \leq 3,1<\beta \leq 2, \varphi_{p}(s)=|s|^{p-2} s$, and $f:[1, e] \times$ $[0,+\infty) \rightarrow[0,+\infty)$ is a positive continuous function. Evidently, for any $p>1, \varphi_{p}^{-1}=\varphi_{q}$, here $1 / p+1 / q=1$. Here $D^{\alpha}$ is the standard Hadamard fractional derivative of order $\alpha$ which is described as follows.

Definition 1 (see [1, Page 111]). The $\alpha$ th Hadamard fractional order derivative of a function $u:[1,+\infty) \mapsto \mathbb{R}$ is defined by

$$
D^{\alpha} u(t)=\frac{1}{\Gamma(n-\alpha)}\left(t \frac{d}{d t}\right)^{n} \int_{1}^{t}\left(\log \frac{t}{s}\right)^{n-\alpha-1} \frac{u(s)}{s} d s,
$$

where $\alpha>0, n=[\alpha]+1$, and $[\alpha]$ denotes the largest integer which is less than or equal to $\alpha$. Correspondingly, the $\alpha$ th Hadamard fractional order integral of $u:[1,+\infty) \mapsto \mathbb{R}$ is defined by

$$
I^{\alpha} u(t)=\frac{1}{\Gamma(\alpha)} \int_{1}^{t}\left(\log \frac{t}{s}\right)^{\alpha-1} \frac{u(s)}{s} d s,
$$

where $\Gamma$ is the gamma function.

To the best of our knowledge, there are few contributions to the Hadamard type with $p$-Laplacian operator; we fill the gap in this paper. In fact, we will discuss the existence and the uniqueness of the positive solutions of (5). The structure of this paper goes on as follows. In Section 2, we will introduce some basic lemmas that will be used. In Section 3, we first give some existence results including Theorems 10 and 11, Corollary 12, and Theorem 13. Then, we will prove Theorems 14 and 15 which reveal the uniqueness of the solution. In Section 4, we give two examples to illustrate our results.

\section{Preliminary Results}

In this section, we will first recall the following preliminary facts that will be used in our main results.

Lemma 2 (see $[1$, Theorem 2.3]). Let $\alpha>0, n=[\alpha]+1$; then

$$
I^{\alpha} D^{\alpha} u(t)=u(t)+\sum_{i=1}^{n} c_{i}(\log t)^{\alpha-i}
$$

where $c_{i}, i=1,2, \ldots, n$, are some constants in $\mathbb{R}$.

The following lemma is the Schauder fixed point theorem which is well known; see Theorem 2.10 in [22].

Lemma 3. If $U$ is a nonempty closed, bounded, and convex subset of a Banach space $X$ and $T: U \rightarrow U$ is completely continuous, then $T$ has a fixed point in $U$.

Lemma 4 (see [24, Lemma 2.7]). Let $X$ be a Banach space, let $P \subseteq X$ be a cone, and let $\Omega_{1}, \Omega_{2}$ be two bounded open balls of E centered at the origin with $\bar{\Omega}_{1} \subset \Omega_{2}$. Suppose that $T: P \cap$ $\left(\bar{\Omega}_{2} \backslash \Omega_{1}\right) \rightarrow P$ is a completely continuous operator such that either

(i) $\|T x\| \leq\|x\|, x \in P \cap \partial \Omega_{1}$ and $\|T x\| \geq\|x\|, x \in P \cap \partial \Omega_{2}$, or

(ii) $\|T x\| \geq\|x\|, x \in P \cap \partial \Omega_{1}$ and $\|T x\| \leq\|x\|, x \in P \cap \partial \Omega_{2}$, holds. Then $T$ has a fixed point in $P \cap\left(\bar{\Omega}_{2} \backslash \Omega_{1}\right)$.

The following conclusion is the nonlinear alternative of Leray-Schauder type; see Lemma 2.6 in [10].

Lemma 5. Let $X$ be a Banach space with $C \subseteq X$ being closed and convex. Assume that $U$ is a relatively open subset of $C$ with $0 \in U$ and $A: \bar{U} \rightarrow C$ is a continuous, compact map. Then either

(1) A has a fixed point in $\bar{U}$, or

(2) there exists $u \in \partial U$ and $\lambda \in(0,1)$, with $u=\lambda A u$.

Next, we give several lemmas which will be applied in the proofs of our main results.

Lemma 6. Let $u(t)$ be the solution of the problem (5); then it can be described as below:

$$
u(t)=\int_{1}^{e} G(t, s) \varphi_{q}\left(\int_{1}^{e} H(s, \tau) f(\tau, u(\tau)) \frac{d \tau}{\tau}\right) \frac{d s}{s}=Y(t)
$$


where

$$
\begin{aligned}
& G(t, s)= \begin{cases}\frac{(\log t)^{\alpha-1}(1-\log s)^{\alpha-2}-(\log (t / s))^{\alpha-1}}{\Gamma(\alpha)}, & s \leq t, \\
\frac{(\log t)^{\alpha-1}(1-\log s)^{\alpha-2}}{\Gamma(\alpha)}, & t \leq s,\end{cases} \\
& H(t, s)= \begin{cases}\frac{(\log t)^{\beta-1}(1-\log s)^{\beta-1}-(\log (t / s))^{\beta-1}}{\Gamma(\beta)}, & s \leq t, \\
\frac{(\log t)^{\beta-1}(1-\log s)^{\beta-1}}{\Gamma(\beta)}, & t \leq s .\end{cases}
\end{aligned}
$$

Proof. Putting $y(t)=f(t, u(t))$, we have $D^{\beta}\left(\varphi_{p}\left(D^{\alpha} u(t)\right)\right)=$ $y(t)$. By Lemma 2 and the fact that $1<\beta \leq 2$,

$$
\varphi_{p}\left(D^{\alpha} u(t)\right)=I^{\beta} y(t)+c_{1}(\log t)^{\beta-1}+c_{2}(\log t)^{\beta-2} .
$$

The boundary value hypotheses give $D^{\alpha} u(1)=D^{\alpha} u(e)=0$. So we can get that

$$
c_{2}=0, \quad c_{1}=-\frac{1}{\Gamma(\beta)} \int_{1}^{e}(1-\log \tau)^{\beta-1} y(\tau) \frac{d \tau}{\tau} .
$$

Therefore,

$$
\begin{aligned}
\varphi_{p}\left(D^{\alpha} u(t)\right) \\
=I^{\beta} y(t)-\frac{(\log t)^{\beta-1}}{\Gamma(\beta)} \int_{1}^{e}(1-\log \tau)^{\beta-1} y(\tau) \frac{d \tau}{\tau} \\
=\frac{1}{\Gamma(\beta)} \int_{1}^{t}\left(\log \frac{t}{\tau}\right)^{\beta-1} y(\tau) \frac{d \tau}{\tau} \\
-\frac{(\log t)^{\beta-1}}{\Gamma(\beta)} \int_{1}^{t}(1-\log \tau)^{\beta-1} y(\tau) \frac{d \tau}{\tau} \\
-\frac{(\log t)^{\beta-1}}{\Gamma(\beta)} \int_{t}^{e}(1-\log \tau)^{\beta-1} y(\tau) \frac{d \tau}{\tau} \\
=-\int_{1}^{t} \frac{(\log t)^{\beta-1}(1-\log \tau)^{\beta-1}-(\log t-\log \tau)^{\beta-1}}{\Gamma(\beta)} \\
\quad \times y(\tau) \frac{d \tau}{\tau}-\int_{t}^{e} \frac{(\log t)^{\beta-1}(1-\log \tau)^{\beta-1}}{\Gamma(\beta)} y(\tau) \frac{d \tau}{\tau} \\
=-\int_{1}^{e} H(t, \tau) y(\tau) \frac{d \tau}{\tau} .
\end{aligned}
$$

Notice the fact that $\varphi_{p}^{-1}=\varphi_{q}, 1 / p+1 / q=1$; we have

$$
D^{\alpha} u(t)+\varphi_{q}\left(\int_{1}^{e} H(t, \tau) f(\tau, u(\tau)) \frac{d \tau}{\tau}\right)=0 .
$$

Putting $x(t)=\varphi_{q}\left(\int_{1}^{e} H(t, \tau) f(\tau, u(\tau))(d \tau / \tau)\right)$, it follows from Lemma 2 that

$u(t)=-I^{\alpha} x(t)+C_{1}(\log t)^{\alpha-1}+C_{2}(\log t)^{\alpha-2}+C_{3}(\log t)^{\alpha-3}$.
This, combined with the fact that $u(1)=u^{\prime}(1)=u^{\prime}(e)=0$, yields

$$
\begin{aligned}
& C_{3}=0, \quad C_{2}=0, \\
& C_{1}=\frac{1}{\Gamma(\alpha)} \int_{1}^{e}(1-\log s)^{\alpha-2} x(s) \frac{d s}{s} .
\end{aligned}
$$

Thus,

$$
\begin{aligned}
u(t) & =\int_{1}^{e} G(t, s) x(s) \frac{d s}{s} \\
& =\int_{1}^{e} G(t, s) \varphi_{q}\left(\int_{1}^{e} H(s, \tau) f(\tau, u(\tau)) \frac{d \tau}{\tau}\right) \frac{d s}{s},
\end{aligned}
$$

where

$$
\begin{aligned}
& G(t, s)= \begin{cases}\frac{(\log t)^{\alpha-1}(1-\log s)^{\alpha-2}-(\log (t / s))^{\alpha-1}}{\Gamma(\alpha)}, & s \leq t, \\
\frac{(\log t)^{\alpha-1}(1-\log s)^{\alpha-2}}{\Gamma(\alpha)}, & t \leq s,\end{cases} \\
& H(t, s)= \begin{cases}\frac{(\log t)^{\beta-1}(1-\log s)^{\beta-1}-(\log (t / s))^{\beta-1}}{\Gamma(\beta)}, & s \leq t, \\
\frac{(\log t)^{\beta-1}(1-\log s)^{\beta-1}}{\Gamma(\beta)}, & t \leq s .\end{cases}
\end{aligned}
$$

This completes the proof of Lemma 6 .

Lemma 7. Suppose that $2<\alpha \leq 3,1<\beta \leq 2$. Then the functions $G(t, s)$ and $H(t, s)$ defined in (10) have the following properties:

(1) $G(t, s), H(t, s)$ are continuous on $[1, e] \times[1, e]$;

(2) for any $t, s \in[1, e], G(t, s) \geq 0, H(t, s) \geq 0$;

(3) for any $t, s \in[1, e], G(t, s) \leq G(e, s), H(t, s) \leq H(s, s)$;

(4) there exist two positive functions $\gamma_{1}, \gamma_{2} \in C(1, e)$ such that

$$
\begin{aligned}
\min _{e^{1 / 4} \leq t \leq e^{3 / 4}} G(t, s) & \geq \gamma_{1}(s) \max _{1 \leq t \leq e} G(t, s) \\
& =\gamma_{1}(s) G(e, s), \quad \text { for } s \in(1, e), \\
\min _{e^{1 / 4} \leq t \leq e^{3 / 4}} H(t, s) & \geq \gamma_{2}(s) \max _{1 \leq t \leq e} H(t, s) \\
& =\gamma_{2}(s) H(s, s), \quad \text { for } s \in(1, e) .
\end{aligned}
$$

Proof. (1) and (2) are evident from the expression of $G(t, s)$ and $H(t, s)$. Since, for any fixed number $s \in[1, e], G(t, s)$ is an 
increasing function on $[1, e]$ and $H(t, s)$ is a decreasing function on $[s, e]$ and increasing on $[1, s]$, we get (3). To prove (4), suppose that

$\gamma_{1}(s)$

$= \begin{cases}\frac{(1 / 4)^{\alpha-1}(1-\log s)^{\alpha-2}-(1 / 4-\log s)^{\alpha-1}}{(1-\log s)^{\alpha-2}-(1-\log s)^{\alpha-1}}, & s \in\left(1, e^{1 / 4}\right], \\ \frac{(1 / 4)^{\alpha-1}(1-\log s)^{\alpha-2}}{(1-\log s)^{\alpha-2}-(1-\log s)^{\alpha-1}}, & s \in\left[e^{1 / 4}, e\right),\end{cases}$

and put

$$
\begin{gathered}
p_{1}(t, s)=\frac{(\log t)^{\alpha-1}(1-\log s)^{\alpha-2}-(\log (t / s))^{\alpha-1}}{\Gamma(\alpha)}, \\
p_{2}(t, s)=\frac{(\log t)^{\alpha-1}(1-\log s)^{\alpha-2}}{\Gamma(\alpha)} .
\end{gathered}
$$

The monotonicity of $G(t, s)$ gives

$$
\begin{aligned}
& \min _{e^{1 / 4} \leq t \leq e^{3 / 4}} G(t, s) \\
& = \begin{cases}p_{1}\left(e^{1 / 4}, s\right), & s \in\left(1, e^{1 / 4}\right], \\
p_{2}\left(e^{1 / 4}, s\right), & s \in\left[e^{1 / 4}, e\right),\end{cases} \\
& =\left\{\begin{array}{rr}
\frac{1}{\Gamma(\alpha)}\left[\left(\frac{1}{4}\right)^{\alpha-1}(1-\log s)^{\alpha-2}\right. & \\
\left.-\left(\frac{1}{4}-\log s\right)^{\alpha-1}\right], & s \in\left(1, e^{1 / 4}\right], \\
\frac{1}{\Gamma(\alpha)}\left(\frac{1}{4}\right)^{\alpha-1}(1-\log s)^{\alpha-2}, & s \in\left[e^{1 / 4}, e\right),
\end{array}\right.
\end{aligned}
$$$$
\max _{1 \leq t \leq e} G(t, s)=G(e, s)
$$$$
=\frac{1}{\Gamma(\alpha)}\left[(1-\log s)^{\alpha-2}-(1-\log s)^{\alpha-1}\right],
$$$$
s \in(1, e) \text {. }
$$

Which implies that (19) holds.

Similarly, by writing

$$
\begin{gathered}
q_{1}(t, s)=\frac{(\log t)^{\beta-1}(1-\log s)^{\beta-1}-(\log (t / s))^{\beta-1}}{\Gamma(\beta)}, \\
q_{2}(t, s)=\frac{(\log t)^{\beta-1}(1-\log s)^{\beta-1}}{\Gamma(\beta)}
\end{gathered}
$$

and applying the monotonicity of $H(t, s)$, we have

$$
\begin{aligned}
& \min _{e^{1 / 4} \leq t \leq e^{3 / 4}} H(t, s) \\
& =\left\{\begin{array}{cl}
q_{1}\left(e^{3 / 4}, s\right), & s \in\left(1, e^{1 / 4}\right], \\
\min \left\{q_{1}\left(e^{3 / 4}, s\right),\right. & \\
\left.q_{2}\left(e^{1 / 4}, s\right)\right\}, & s \in\left[e^{1 / 4}, e^{3 / 4}\right], \\
q_{2}\left(e^{1 / 4}, s\right), & s \in\left[e^{3 / 4}, e\right),
\end{array}\right. \\
& = \begin{cases}q_{1}\left(e^{3 / 4}, s\right), & s \in(1, r], \\
q_{2}\left(e^{1 / 4}, s\right), & s \in[r, e),\end{cases}
\end{aligned}
$$

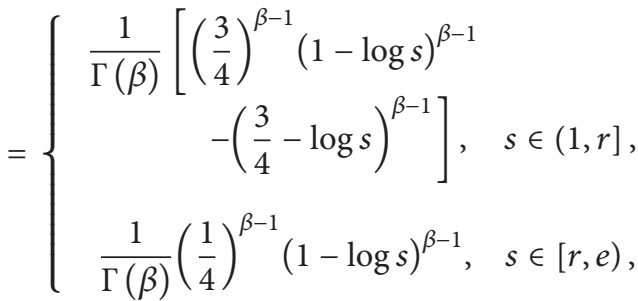

$$
\begin{aligned}
& \max _{1 \leq t \leq e} H(t, s)=H(s, s) \\
& =\frac{1}{\Gamma(\beta)}[\log s(1-\log s)]^{\beta-1}, \\
& s \in(1, e),
\end{aligned}
$$

where $e^{1 / 4}<r<e^{3 / 4}$ is the unique solution of the equation

$$
\left[\frac{3}{4}(1-\log s)\right]^{\beta-1}-\left(\frac{3}{4}-\log s\right)^{\beta-1}=\left[\frac{1}{4}(1-\log s)\right]^{\beta-1} \text {. }
$$

Hence, setting

$\gamma_{2}(s)$

$$
= \begin{cases}\frac{[(3 / 4)(1-\log s)]^{\beta-1}-(3 / 4-\log s)^{\beta-1}}{[\log s(1-\log s)]^{\beta-1}}, & s \in(1, r], \\ \frac{1}{(4 \log s)^{\beta-1}}, & s \in[r, e),\end{cases}
$$

we obtain (20). This completes the proof of Lemma 7.

Let $X=C[1, e],\|u\|=\max _{1 \leq t \leq e}|u(t)|$. we define the cone $P=\{u \in X \mid u(t) \geq 0\}$ and the bounded closed set $U=\{u \in$ $X \mid 0 \leq u(t) \leq K\}$.

The operator $T: X \rightarrow X$ is defined as the following form: $T u(t)=Y(t)$

$$
=\int_{1}^{e} G(t, s) \varphi_{q}\left(\int_{1}^{e} H(s, \tau) f(\tau, u(\tau)) \frac{d \tau}{\tau}\right) \frac{d s}{s} .
$$


Evidently, the solutions of boundary value problem (5) are the corresponding fixed points of the operator $T$.

Lemma 8. Suppose that $T: P \rightarrow X$ is an operator as above; then $T: P \rightarrow P$ is completely continuous.

Proof. It is easy to see that $T: P \rightarrow P$ is continuous. Let $\Omega \subset P$ be a bounded set; then there is a positive constant $A>0$ such that $\|u\| \leq A$ for any $u \in \Omega$. Write $B=$ $\max _{1 \leq t \leq e, 0 \leq u \leq A} f(t, u(t))+1$. For any $u \in \Omega$, we have

$$
\begin{aligned}
|T u(t)| & =\int_{1}^{e} G(t, s) \varphi_{q}\left(\int_{1}^{e} H(s, \tau) f(\tau, u(\tau)) \frac{d \tau}{\tau}\right) \frac{d s}{s} \\
& \leq B^{q-1} \int_{1}^{e} G(e, s) \frac{d s}{s} \varphi_{q}\left(\int_{1}^{e} H(s, \tau) f(\tau, u(\tau))\right) \frac{d \tau}{\tau} \\
& <+\infty
\end{aligned}
$$

which shows that $T \Omega$ is uniformly bounded.

Next, the continuity of $G(t, s)$ implies that, for any $\varepsilon>0$, there exists a constant $\delta$ such that, for any $t_{1}, t_{2} \in[1, e]$, if $\left|t_{1}-t_{2}\right|<\delta$, then

$$
\left|G\left(t_{1}, s\right)-G\left(t_{2}, s\right)\right|<\frac{\varepsilon}{B^{q-1} \varphi_{q}\left(\int_{1}^{e} H(\tau, \tau)(d \tau / \tau)\right)} .
$$

Therefore, for any $u \in \Omega$,

$$
\begin{aligned}
\mid T u\left(t_{1}\right)- & T u\left(t_{2}\right) \mid \\
= & \mid \int_{1}^{e}\left(G\left(t_{1}, s\right)-G\left(t_{2}, s\right)\right) \\
& \quad \times \varphi_{q}\left(\int_{1}^{e} H(s, \tau) f(\tau, u(\tau)) \frac{d \tau}{\tau}\right) \frac{d s}{s} \mid \\
\leq & B^{q-1} \int_{1}^{e}\left|G\left(t_{1}, s\right)-G\left(t_{2}, s\right)\right| \\
& \times \varphi_{q}\left(\int_{1}^{e} H(\tau, \tau) \frac{d \tau}{\tau}\right) \frac{d s}{s}<\varepsilon .
\end{aligned}
$$

That is, $T \Omega$ is equicontinuity. By the means of Arzela-Ascoli theorem [26], we have that $T: P \rightarrow P$ is completely continuous. This completes the proof of Lemma 8.

In the final part of this section, we list the following basic properties of the $p$-Laplacian operator.

Lemma 9. (1) If $1<p<2, x y>0$, and $|x|,|y| \geq m>0$, then

$$
\left|\varphi_{p}(x)-\varphi_{p}(y)\right| \leq(p-1) m^{p-2}|x-y| .
$$

(2) If $p>2,|x|,|y| \leq M$, then

$$
\left|\varphi_{p}(x)-\varphi_{p}(y)\right| \leq(p-1) M^{p-2}|x-y| .
$$

\section{Proofs of the Main Results}

In this section, first, we consider the existence of the solutions of problem (5).
Theorem 10. If $\left[\max _{1 \leq t \leq e, 0 \leq u \leq K} f(t, u)\right]^{q-1} \int_{1}^{e} G(e, s)(d s / s) \varphi_{q}$ $\left(\int_{1}^{e} H(\tau, \tau)(d \tau / \tau)\right) \leq K$, then the boundary value problem $(5)$ has at least one positive solution.

Proof. For any $u \in U$, by the assumption as above and the nonnegativeness of $G(t, s), H(t, s)$, and $f(t, u)$, we have

$$
\begin{aligned}
0 \leq T u(t)= & \int_{1}^{e} G(t, s) \varphi_{q}\left(\int_{1}^{e} H(s, \tau) f(\tau, u(\tau)) \frac{d \tau}{\tau}\right) \frac{d s}{s} \\
\leq & {\left[\max _{1 \leq t \leq e, 0 \leq u \leq K} f(t, u)\right]^{q-1} } \\
& \times \int_{1}^{e} G(e, s) \frac{d s}{s} \varphi_{q}\left(\int_{1}^{e} H(\tau, \tau) \frac{d \tau}{\tau}\right) \\
\leq & K .
\end{aligned}
$$

Therefore, $T$ is a mapping from $U$ to $U$. This, combined with the continuity of $G(t, s), H(t, s)$, and $f(t, u)$, implies that $T$ : $U \rightarrow U$ is continuous.

Let $\Omega \subset U$ be a bounded set; then there exists a positive constant $A$ such that $\|u\| \leq A$ for any $u \in \Omega$. So we have, for any $u \in \Omega$,

$$
\begin{aligned}
|T u(t)|= & \int_{1}^{e} G(t, s) \varphi_{q}\left(\int_{1}^{e} H(s, \tau) f(\tau, u(\tau)) \frac{d \tau}{\tau}\right) \frac{d s}{s} \\
\leq & {\left[\max _{1 \leq t \leq e, 0 \leq u \leq A} f(t, u)\right]^{q-1} } \\
& \times \int_{1}^{e} G(e, s) \frac{d s}{s} \varphi_{q}\left(\int_{1}^{e} H(\tau, \tau) \frac{d \tau}{\tau}\right) \\
\leq & {\left[\max _{1 \leq t \leq e, 0 \leq u \leq K} f(t, u)\right]^{q-1} } \\
& \times \int_{1}^{e} G(e, s) \frac{d s}{s} \varphi_{q}\left(\int_{1}^{e} H(\tau, \tau) \frac{d \tau}{\tau}\right) \leq K \leq+\infty .
\end{aligned}
$$

Therefore, $T \Omega$ is uniformly bounded.

Since $G(t, s)$ is continuous, for any $\varepsilon>0$, there exists a constant $\delta>0$ satisfying that, for any $t_{1}, t_{2} \in[1, e]$ and $\left|t_{1}-t_{2}\right|<\delta$,

$$
\left|G\left(t_{1}, s\right)-G\left(t_{2}, s\right)\right|
$$

$$
<\frac{\varepsilon}{\left[\max _{1 \leq t \leq e, 0 \leq u \leq A} f(t, u)+1\right]^{q-1} \varphi_{q}\left(\int_{1}^{e} H(\tau, \tau)(d \tau / \tau)\right)} .
$$


Then, for any $u \in \Omega$,

$$
\begin{aligned}
\left|T u\left(t_{1}\right)-T u\left(t_{2}\right)\right| \leq & \int_{1}^{e}\left|G\left(t_{1}, s\right)-G\left(t_{2}, s\right)\right| \\
& \times \varphi_{q}\left(\int_{1}^{e} H(s, \tau) f(\tau, u(\tau)) \frac{d \tau}{\tau}\right) \frac{d s}{s} \\
\leq & {\left[\max _{1 \leq t \leq e, 0 \leq u \leq A} f(t, u)+1\right]^{q-1} } \\
& \times \varphi_{q}\left(\int_{1}^{e} H(\tau, \tau) \frac{d \tau}{\tau}\right) \\
& \times \int_{1}^{e}\left|G\left(t_{1}, s\right)-G\left(t_{2}, s\right)\right| \frac{d s}{s}<\varepsilon
\end{aligned}
$$

which shows that $T \Omega$ is equicontinuous. By Arzela-Ascoli theorem [26], $T: U \rightarrow U$ is a completely continuous operator. It follows from Lemma 3 that $T$ has a fixed point $u$ in $U$. That is, problem (5) has at least one positive solution. This completes the proof of Theorem 10.

Let us denote

$$
\begin{aligned}
\Lambda_{1}= & \left(\int_{e^{1 / 4}}^{e^{3 / 4}} \gamma_{1}(s) G(e, s) \frac{d s}{s}\right. \\
& \left.\times \varphi_{q}\left(\int_{e^{1 / 4}}^{e^{3 / 4}} \gamma_{2}(\tau) H(\tau, \tau) \frac{d \tau}{\tau}\right)\right)^{-1}, \\
\Lambda_{2}= & \left(\int_{1}^{e} G(e, s) \frac{d s}{s} \varphi_{q}\left(\int_{1}^{e} H(\tau, \tau) \frac{d \tau}{\tau}\right)\right)^{-1} .
\end{aligned}
$$

Theorem 11. Suppose that $f(t, u):[1, e] \times[0,+\infty)$ is a continuous function and there exist two constants $r_{2}>r_{1}>0$ satisfying that

(i) $f(t, u) \geq \varphi_{p}\left(\Lambda_{1} r_{1}\right)$, for $(t, u) \in[1, e] \times\left[0, r_{1}\right]$;

(ii) $f(t, u) \leq \varphi_{p}\left(\Lambda_{2} r_{2}\right)$, for $(t, u) \in[1, e] \times\left[0, r_{2}\right]$.

Then the boundary value problem (5) has at least one positive solution $u$ which satisfies that $r_{1} \leq\|u\| \leq r_{2}$.
Proof. Let $\Omega_{1}=\left\{u \in P \mid\|u\|<r_{1}\right\}$. For any $u \in \partial \Omega_{1}$, we have $0 \leq u(t) \leq r_{1}$ for $t \in[1, e]$. By the assumption (i), for any $t \in\left[e^{1 / 4}, e^{3 / 4}\right]$,

$$
\begin{aligned}
|T u(t)|= & \int_{1}^{e} G(t, s) \\
& \times \varphi_{q}\left(\int_{1}^{e} H(s, \tau) f(\tau, u(\tau)) \frac{d \tau}{\tau}\right) \frac{d s}{s} \\
\geq & \Lambda_{1} r_{1} \int_{1}^{e} G(t, s) \varphi_{q}\left(\int_{1}^{e} H(s, \tau) \frac{d \tau}{\tau}\right) \frac{d s}{s} \\
\geq & \Lambda_{1} r_{1} \int_{e^{1 / 4}}^{e^{3 / 4}} G(t, s) \varphi_{q}\left(\int_{e^{1 / 4}}^{e^{3 / 4}} H(s, \tau) \frac{d \tau}{\tau}\right) \frac{d s}{s} \\
\geq & \Lambda_{1} r_{1} \int_{e^{1 / 4}}^{e^{3 / 4}} \gamma_{1}(s) G(e, s) \\
& \times \varphi_{q}\left(\int_{e^{1 / 4}}^{e^{3 / 4}} \gamma_{2}(\tau) H(\tau, \tau) \frac{d \tau}{\tau}\right) \frac{d s}{s}=r_{1}=\|u\| .
\end{aligned}
$$

Hence,

$$
\|T u\| \geq\|u\|, \quad \text { for } u \in \partial \Omega_{1}
$$

Similarly, let $\Omega_{2}:=\left\{u \in P \mid\|u\|<r_{2}\right\}$. For any $u \in \partial \Omega_{2}$, we get $0 \leq u(t) \leq r_{2}, t \in[1, e]$. It follows from (ii) that for any $t \in[1, e]$,

$$
\begin{aligned}
|T u(t)| & =\int_{1}^{e} G(t, s) \varphi_{q}\left(\int_{1}^{e} H(s, \tau) f(\tau, u(\tau)) \frac{d \tau}{\tau}\right) \frac{d s}{s} \\
& \leq \Lambda_{2} r_{2} \int_{1}^{e} G(t, s) \varphi_{q}\left(\int_{1}^{e} H(s, \tau) \frac{d \tau}{\tau}\right) \frac{d s}{s} \\
& \leq \Lambda_{2} r_{2} \int_{1}^{e} G(e, s) \varphi_{q}\left(\int_{1}^{e} H(\tau, \tau) \frac{d \tau}{\tau}\right) \frac{d s}{s} \\
& =r_{2}=\|u\| .
\end{aligned}
$$

Therefore,

$$
\|T u\| \leq\|u\|, \quad \text { for } u \in \partial \Omega_{2} .
$$

By Lemmas 4 and $8, T$ has a fixed point in $\bar{\Omega}_{2} \backslash \Omega_{1}$. Therefore, the boundary value problem (5) has one positive solution in $\bar{\Omega}_{2} \backslash \Omega_{1}$. This completes the proof of Theorem 11 .

Corollary 12. Suppose that $f(t, u):[1, e] \times[0,+\infty)$ is a continuous function and there exist two constants $r_{2}>r_{1}>0$ satisfying that

(i) $f(t, u) \leq \varphi_{p}\left(\Lambda_{2} r_{1}\right)$, for $(t, u) \in[1, e] \times\left[0, r_{1}\right]$;

(ii) $f(t, u) \geq \varphi_{p}\left(\Lambda_{1} r_{2}\right)$, for $(t, u) \in[1, e] \times\left[0, r_{2}\right]$.

Then the boundary value problem (5) has at least one positive solution $u$ which satisfies that $r_{1} \leq\|u\| \leq r_{2}$. 
Proof. The proof of Corollary 12 is similar to the one of Theorem 11. So we omit the detail.

Theorem 13. Suppose that $f(t, u):[1, e] \times[0,+\infty)$ is a positive continuous function and there exists a constant $r>0$ such that

$$
\begin{aligned}
r> & {\left[\max _{1 \leq t \leq e, 0 \leq u \leq r} f(t, u)+1\right]^{q-1} } \\
& \times \int_{1}^{e} G(e, s) \frac{d s}{s} \varphi_{q}\left(\int_{1}^{e} H(\tau, \tau) \frac{d \tau}{\tau}\right) .
\end{aligned}
$$

Then the boundary value problem (5) has at least one positive solution.

Proof. Let

$$
E=\{u \in P:\|u\|<r\} .
$$

From Lemma 8, we know $T: \bar{E} \rightarrow P$ is completely continuous. Assume that there exist $u \in \bar{E}, \lambda \in(0,1)$ such that $u=\lambda T u$. Then we have

$$
\begin{aligned}
|u(t)|= & |\lambda T u| \\
\leq & \left|\int_{1}^{e} G(t, s) \varphi_{q}\left(\int_{1}^{e} H(s, \tau) f(\tau, u(\tau)) \frac{d \tau}{\tau}\right) \frac{d s}{s}\right| \\
\leq & {\left[\max _{1 \leq t \leq e, 0 \leq u \leq r} f(t, u)+1\right]^{q-1} } \\
& \times \int_{1}^{e} G(e, s) \frac{d s}{s} \varphi_{q}\left(\int_{1}^{e} H(\tau, \tau) \frac{d \tau}{\tau}\right) .
\end{aligned}
$$

Thus,

$$
\begin{aligned}
\|u\| \leq & {\left[\max _{1 \leq t \leq e, 0 \leq u \leq r} f(t, u)+1\right]^{q-1} } \\
& \times \int_{1}^{e} G(e, s) \frac{d s}{s} \varphi_{q}\left(\int_{1}^{e} H(\tau, \tau) \frac{d \tau}{\tau}\right) .
\end{aligned}
$$

By (43), we can imply that $\|u\|<r$, which means that $u \notin$ $\partial E$. That is to say, there is no $u \in \partial E$ such that $u=\lambda T u$ for some $\lambda \in(0,1)$. Therefore, by Lemma 5 , we conclude that the problem (5) has at least one positive solution. This completes the proof of Theorem 13.

Now we turn to the uniqueness of solution for boundary value problem (5).

Theorem 14. Suppose that $p>2$. If there exists a nonnegative function $g$ satisfying that

(1) for any $(t, u) \in[1, e] \times[0,+\infty), f(t, u) \geq g(t)$;

(2) $N=\int_{e^{1 / 4}}^{e^{3 / 4}} \gamma_{2}(\tau) H(\tau, \tau) g(\tau)(d \tau / \tau)>0$;

(3) for any $t \in[1, e], u, v \in[0,+\infty),|f(t, u)-f(t, v)| \leq$ $L|u-v|$, where

$$
0<L<\left(\int_{1}^{e} G(e, s) \frac{d s}{s} \int_{1}^{e} H(\tau, \tau) \frac{d \tau}{\tau}(q-1) N^{q-2}\right)^{-1},
$$

then the boundary value problem (5) has a unique solution.
Proof. Assume that $u, v$ are two positive solutions of problem (5). It is easy to see that

$$
\begin{aligned}
& \int_{1}^{e} H(s, \tau) f(\tau, u(\tau)) \frac{d \tau}{\tau} \\
& \quad \geq \int_{e^{1 / 4}}^{e^{3 / 4}} \gamma_{2}(\tau) H(\tau, \tau) g(\tau) \frac{d \tau}{\tau}=N>0 ;
\end{aligned}
$$

then by the fact $p>2$ (i.e., its dual exponent $1<q<2$ ) and Lemma 9, we have

$$
\begin{aligned}
|u(t)-v(t)| \leq & \int_{1}^{e} G(t, s)(q-1) N^{q-2} \\
& \times \mid \int_{1}^{e} H(s, \tau) f(\tau, u(\tau)) \frac{d \tau}{\tau} \\
& -\int_{1}^{e} H(s, \tau) f(\tau, v(\tau)) \frac{d \tau}{\tau} \mid \frac{d s}{s} \\
\leq & \int_{1}^{e} G(t, s)(q-1) N^{q-2} \\
& \times \int_{1}^{e} H(s, \tau)|f(\tau, u(\tau))-f(\tau, v(\tau))| \frac{d \tau}{\tau} \frac{d s}{s} \\
\leq & \int_{1}^{e} G(e, s)(q-1) N^{q-2} \\
& \times \int_{1}^{e} H(\tau, \tau) L\|u-v\| \frac{d \tau}{\tau} \frac{d s}{s} \\
= & \int_{1}^{e} G(e, s) \frac{d s}{s} \\
& \times \int_{1}^{e} H(\tau, \tau) \frac{d \tau}{\tau}(q-1) N^{q-2} L\|u-v\| \\
= & \delta_{1}\|u-v\|,
\end{aligned}
$$

where $\delta_{1}=\int_{1}^{e} G(e, s)(d s / s) \int_{1}^{e} H(\tau, \tau)(d \tau / \tau)(q-1) N^{q-2} L$. So we can get

$$
\|u-v\| \leq \delta_{1}\|u-v\| .
$$

By the third hypothesis, $0<\delta_{1}<1$, which implies that $u(t)=$ $v(t)$. And this completes the proof of Theorem 14 .

By using the same way, we can prove the last one of our main uniqueness results.

Theorem 15. Suppose that $1<p<2$. If there exists a nonnegative function $h$ satisfying that

(1) for any $(t, u) \in[1, e] \times[0,+\infty), f(t, u) \leq h(t)$; 
(2) $M=\int_{1}^{e} H(\tau, \tau) h(\tau)(d \tau / \tau)>0$;

(3) for any $t \in[1, e], u, v \in[0,+\infty),|f(t, u)-f(t, v)| \leq$ $L|u-v|$, where

$0<L<\left(\int_{1}^{e} G(e, s) \frac{d s}{s} \int_{1}^{e} H(\tau, \tau) \frac{d \tau}{\tau}(q-1) M^{q-2}\right)^{-1}$,

then the boundary value problem (5) has a unique solution.

\section{Examples}

In this section we give several examples to illustrate our main results.

Example 16. Consider the boundary value problem:

$$
\begin{gathered}
D^{3 / 2}\left(\varphi_{5 / 2}\left(D^{5 / 2} u(t)\right)\right) \\
=(1-\log t)^{1 / 2}\left(1+\sin ^{2} u\right), \quad 1<t<e, \\
u(1)=u^{\prime}(1)=u^{\prime}(e)=0, \\
D^{5 / 2} u(1)=D^{5 / 2} u(e)=0 .
\end{gathered}
$$

Then the boundary value problem has a unique positive solution.

Proof. Since $\alpha=5 / 2, \beta=3 / 2$, a straightforward calculation gives

$$
\begin{aligned}
\int_{1}^{e} G(e, s) \frac{d s}{s}= & \int_{1}^{e} \frac{(1-\log s)^{\alpha-2}-(1-\log s)^{\alpha-1}}{\Gamma(\alpha)} \frac{d s}{s} \\
= & \frac{1}{\Gamma(\alpha)} \int_{1}^{e}(1-\log s)^{\alpha-2} \frac{d s}{s} \\
& -\frac{1}{\Gamma(\alpha)} \int_{1}^{e}(1-\log s)^{\alpha-1} \frac{d s}{s} \\
= & \frac{1}{\Gamma(\alpha)} \int_{0}^{1}(1-t)^{\alpha-2} d t \\
& -\frac{1}{\Gamma(\alpha)} \int_{0}^{1}(1-t)^{\alpha-1} d t \\
= & \frac{1}{(\alpha-1) \Gamma(\alpha+1)} \\
= & \frac{1}{(3 / 2) \Gamma(7 / 2)}=\frac{16}{45 \sqrt{\pi}},
\end{aligned}
$$

$$
\begin{aligned}
\int_{1}^{e} H(\tau, \tau) \frac{d \tau}{\tau} & =\int_{1}^{e} \frac{(\log \tau)^{\beta-1}(1-\log \tau)^{\beta-1}}{\Gamma(\beta)} \frac{d \tau}{\tau} \\
& =\frac{1}{\Gamma(\beta)} \int_{0}^{1} t^{\beta-1}(1-t)^{\beta-1} d t \\
& =\frac{\Gamma(\beta)}{\Gamma(2 \beta)} \\
& =\frac{\Gamma(3 / 2)}{\Gamma(3)} \\
& =\frac{\sqrt{\pi}}{4} .
\end{aligned}
$$

Taking $K=1, f(t, u)=(1-\log t)^{1 / 2}\left(1+\sin ^{2} u\right)$, and $p=5 / 2>2$ (its dual exponent $q=5 / 3$ ), we have

$$
\begin{aligned}
& \max _{1 \leq t \leq e, 0 \leq u \leq 1} f(t, u)=1+\sin ^{2} 1<2, \\
& {\left[\max _{1 \leq t \leq e, 0 \leq u \leq 1} f(t, u)\right]^{(5 / 3)-1} \int_{1}^{e} G(e, s) \frac{d s}{s} \varphi_{5 / 3}\left(\int_{1}^{e} H(\tau, \tau) \frac{d \tau}{\tau}\right)} \\
& \quad<2^{2 / 3} \cdot \frac{16}{45 \sqrt{\pi}} \cdot\left(\frac{\sqrt{\pi}}{4}\right)^{2 / 3} \approx 0.185<1 .
\end{aligned}
$$

By Theorem 10, the boundary value problem (52) has at least one positive solution.

Choosing the nonnegative function $g(t)=(1-\log t)^{1 / 2}$, for any $(t, u) \in[1, e] \times[0,+\infty)$, we gain that $f(t, u)=(1-$ $\log t)^{1 / 2}\left(1+\sin ^{2} u\right) \geq g(t)$. Then

$$
\begin{aligned}
N= & \int_{e^{1 / 4}}^{e^{3 / 4}} \gamma_{2}(\tau) H(\tau, \tau) g(\tau) \frac{d \tau}{\tau} \\
= & \int_{e^{1 / 4}}^{r} \frac{1}{\Gamma(3 / 2)}\left\{\left[\frac{3}{4}(1-\log \tau)\right]^{3 / 2-1}\right. \\
& \left.\quad-\left(\frac{3}{4}-\log \tau\right)^{3 / 2-1}\right\} \cdot(1-\log \tau)^{1 / 2} \frac{d \tau}{\tau} \\
& +\int_{r}^{e^{3 / 4}} \frac{1}{\Gamma(3 / 2)}\left(\frac{1}{4}\right)^{3 / 2-1}(1-\log \tau)^{3 / 2-1} \\
& \cdot(1-\log \tau)^{1 / 2} \frac{d \tau}{\tau} \\
\geq & \int_{r}^{e^{3 / 4}} \frac{1}{\sqrt{\pi}}(1-\log \tau) \frac{d \tau}{\tau} \\
= & \frac{1}{2 \sqrt{\pi}}(1-\log r)^{2}-\frac{1}{32 \sqrt{\pi}}
\end{aligned}
$$




$$
\begin{aligned}
& =\frac{1}{2 \sqrt{\pi}}\left(1-1+\frac{\sqrt{3}}{6}\right)^{2}-\frac{1}{32 \sqrt{\pi}} \\
& =\frac{1}{96 \sqrt{\pi}},
\end{aligned}
$$

where $r=e^{1-(\sqrt{3} / 6)}$ is the solution of (26) when $\beta=3 / 2$. For any $t \in[1, e], u, v \in[0,+\infty)$, taking $L=2$, we obtain

$$
\begin{aligned}
|f(t, u)-f(t, v)| & =(1-\log t)^{1 / 2}\left|\sin ^{2} u-\sin ^{2} v\right| \\
& \leq 2|u-v|=L|u-v| .
\end{aligned}
$$

Thus,

$$
\begin{aligned}
\left(\int_{1}^{e} G\right. & \left.(e, s) \frac{d s}{s} \int_{1}^{e} H(\tau, \tau) \frac{d \tau}{\tau}(q-1) N^{q-2}\right)^{-1} \\
& =\left(\frac{16}{45 \sqrt{\pi}} \cdot \frac{\sqrt{\pi}}{4} \cdot \frac{2}{3} N^{-1 / 3}\right)^{-1} \\
& \geq \frac{135}{8}\left(\frac{1}{96 \sqrt{\pi}}\right)^{1 / 3} \\
& \approx 3.045>2=L .
\end{aligned}
$$

By Theorem 14, the boundary value problem (52) has a unique solution.

Example 17. Consider the following nonlinear boundary value problem:

$$
\begin{gathered}
D^{3 / 2}\left(\varphi_{3 / 2}\left(D^{5 / 2} u(t)\right)\right)=\log ^{1 / 2} t \sin ^{2} u, \quad 1<t<e, \\
u(1)=u^{\prime}(1)=u^{\prime}(e)=0, \quad D^{5 / 2} u(1)=D^{5 / 2} u(e)=0 .
\end{gathered}
$$

Then the boundary value problem has a unique positive solution.

Proof. Taking $r=1$, since $\alpha=5 / 2, \beta=3 / 2, p=3 / 2<2$ (its dual exponent $q=3$ ) and $f(t, u)=\log ^{1 / 2} t \sin ^{2} u$, we obtain

$$
\begin{gathered}
\max _{1 \leq t \leq e, 0 \leq u \leq 1} f(t, u)=\sin ^{2} 1<1 \\
{\left[\max _{1 \leq t \leq e, 0 \leq u \leq 1} f(t, u)+1\right]^{3-1} \int_{1}^{e} G(e, s) \frac{d s}{s} \varphi_{3}\left(\int_{1}^{e} H(\tau, \tau) \frac{d \tau}{\tau}\right)} \\
<4 \cdot \frac{16}{45 \sqrt{\pi}} \cdot \frac{\pi}{16} \approx 0.158<1
\end{gathered}
$$

By means of Theorem 13, the boundary value problem (58) has at least one positive solution.
Taking the nonnegative function $h(t)=\log ^{1 / 2} t$, for $(t, u) \in[1, e] \times[0,+\infty)$, it is easy to obtain $f(t, u)=\log ^{1 / 2} t$ $\sin ^{2} u \leq h(t)$ and

$$
\begin{aligned}
M & =\int_{1}^{e} H(\tau, \tau) h(\tau) \frac{d \tau}{\tau} \\
& =\frac{1}{\Gamma(3 / 2)} \int_{1}^{e} \log \tau(1-\log \tau)^{1 / 2} \frac{d \tau}{\tau}=\frac{8}{15 \sqrt{\pi}} .
\end{aligned}
$$

Choosing $L=2$, for any $t \in[1, e], u, v \in[0,+\infty)$, we have

$$
\begin{aligned}
&|f(t, u)-f(t, v)|=\log ^{1 / 2} t\left|\sin ^{2} u-\sin ^{2} v\right| \\
& \leq 2|u-v|=L|u-v| \\
&\left(\int_{1}^{e} G(e, s) \frac{d s}{s} \int_{1}^{e} H(\tau, \tau) \frac{d \tau}{\tau}(q-1) M^{q-2}\right)^{-1} \\
&=\left(\frac{16}{45 \sqrt{\pi}} \cdot \frac{\sqrt{\pi}}{4} \cdot 2 \cdot \frac{8}{15 \sqrt{\pi}}\right)^{-1} \\
& \approx 18.694>2=L .
\end{aligned}
$$

From Theorem 15, the boundary value problem (58) has a unique solution.

\section{Acknowledgments}

The authors would like to thank the referees for their detailed and helpful suggestions for revising this paper. This work was supported by the National Natural Science Foundation of China (Grant nos. 11226167 and 11361020), the Natural Science Foundation of Hainan Province (no. 111005), and the Ph.D. Scientific Research Starting Foundation of Hainan Normal University (no. HSBS1016).

\section{References}

[1] A. A. Kilbas, H. M. Srivastava, and J. J. Trujillo, Theory and Applications of Fractional Differential Equations, Elsevier, Amsterdam, The Netherlands, 2006.

[2] K. S. Miller and B. Ross, An Introduction to the Fractional Calculus and Fractional Differential Equation, Wiley, New York, NY, USA, 1993.

[3] I. Podlubny, Fractional Differential Equations, Academic Press, New York, NY, USA, 1999.

[4] J. Sabatier, O. P. Agrawal, and J. T. Machado, Advances in Fractional Calculus: Theoretical Developments and Applications in Physics and Engineering, Springer, Dordrecht, The Netherlands, 2007.

[5] B. Ahmad, S. K. Ntouyas, and A. Alsaedi, "New existence results for nonlinear fractional differential equations with threepoint integral boundary conditions," Advances in Difference Equations, vol. 2011, Article ID 107384, 11 pages, 2011.

[6] R. P. Agarwal, D. O’Regan, and S. Staněk, "Positive solutions for Dirichlet problems of singular nonlinear fractional differential equations," Journal of Mathematical Analysis and Applications, vol. 371, no. 1, pp. 57-68, 2010.

[7] A. Babakhani and V. Daftardar-Gejji, "Existence of positive solutions of nonlinear fractional differential equations," Journal of 
Mathematical Analysis and Applications, vol. 278, no. 2, pp. 434442, 2003.

[8] M. Benchohra, S. Hamani, and S. K. Ntouyas, "Boundary value problems for differential equations with fractional order and nonlocal conditions," Nonlinear Analysis, Theory, Methods and Applications, vol. 71, no. 7-8, pp. 2391-2396, 2009.

[9] Z. Bai and H. Lü, "Positive solutions for boundary value problem of nonlinear fractional differential equation," Journal of Mathematical Analysis and Applications, vol. 311, no. 2, pp. 495505, 2005.

[10] A. Chen and Y. Chen, "Existence of solutions to anti-periodic boundary value problem for nonlinear fractional differential equations with impulses," Advances in Difference Equations, vol. 2011, Article ID 915689, 17 pages, 2011.

[11] K. Furati and N. Tatar, "An existence resulult for nonlacal fractional differential problem," Journal of Fractional Calculus and Applications, vol. 26, pp. 43-51, 2004.

[12] X. Liu and M. Jia, "Multiple solutions for fractional differential equations with nonlinear boundary conditions," Computers and Mathematics with Applications, vol. 59, no. 8, pp. 2880-2886, 2010.

[13] X. Liu, M. Jia, and B. Wu, "Existence and uniqueness of solution for fractional differential equations with integral boundary conditions," Electronic Journal of Qualitative Theory of Differential Equations, vol. 69, pp. 1-10, 2009.

[14] F. Li, J. Liang, T. Lu, and H. Zhu, "A nonlocal Cauchy problem for fractional integrodifferential equations," Journal of Applied Mathematics, vol. 2012, Article ID 901942, 18 pages, 2012.

[15] Z. Lv, J. Liang, and T. Xiao, "Solutions to the Cauchy problem for differential equations in Banach spaces with fractional order," Computers and Mathematics with Applications, vol. 62, no. 3, pp. 1303-1311, 2011.

[16] Z. Lv, J. Liang, and T. Xiao, "Solutions to fractional differential equations with nonlocal initial condition in Banach spaces," Advances in Difference Equations, vol. 2010, Article ID 340349, 10 pages, 2010.

[17] F. Li, T. Xiao, and H. Xu, "On nonlinear neutral fractional integrodifferential inclusions with infinite delay," Journal of Applied Mathematics, vol. 2012, Article ID 916543, 19 pages, 2012.

[18] J. Liang, S. Yan, F. Li, and T. Huang, "On the existence of mild solutions to the Cauchy problem for a class of fractional evolution equation," Advances in Difference Equations, vol. 2012, 40 pages, 2012.

[19] R. Wang, T. Xiao, and J. Liang, "A note on the fractional Cauchy problems with nonlocal initial conditions," Applied Mathematics Letters, vol. 24, no. 8, pp. 1435-1442, 2011.

[20] G. Chai, "Positive solutions for boundary value problem of fractional differential equation with p-Laplacian operator," Boundary Value Problems, vol. 2012, 18 pages, 2012.

[21] T. Chen and W. Liu, "An anti-periodic boundary value problem for fractional differential equation with $p$-Laplacian operator," Applied Mathematics Letters, vol. 25, no. 11, pp. 1671-1675, 2012.

[22] Z. Han, H. Lu, S. Sun, and D. Yang, "Positive solutions to boundary value problems of $p$-Laplacian fractional differential equations with a parameter in the boundary conditions," Electronic Journal of Qualitative Theory of Differential Equations, vol. 2012, no. 213, pp. 1-14, 2012.

[23] X. Liu, M. Jia, and X. Xiang, "On the solvability of a fractional differential equation model involving the $p$-Laplacian operator," Computers and Mathematics with Applications, vol. 64, no. 10, pp. 3267-3275, 2012.
[24] H. Lu, Z. Han, S. Sun, and J. Liu, "Existence on positive solutions for boundary value problems of nonlinear fractional differential equations with $p$-Laplacian," Advances in Difference Equations, vol. 2013, 30 pages, 2013.

[25] J. Wang, H. Xiang, and Z. Liu, "Existence of concave positive solutions for boundary value problem of nonlinear fractional differential equation with $p$-Laplacian operator," International Journal of Mathematics and Mathematical Sciences, vol. 2010, 17 pages, 2010.

[26] K. Yosida, Functional Analisis, Springer, New York, NY, USA, 1999. 


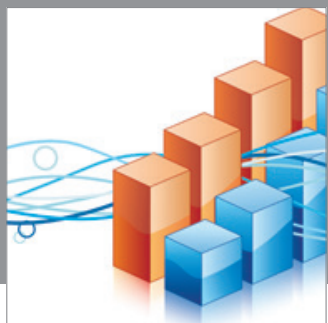

Advances in

Operations Research

mansans

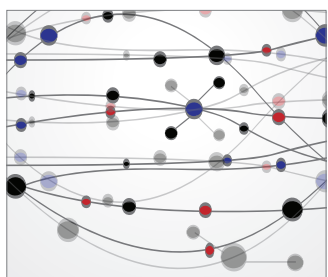

The Scientific World Journal
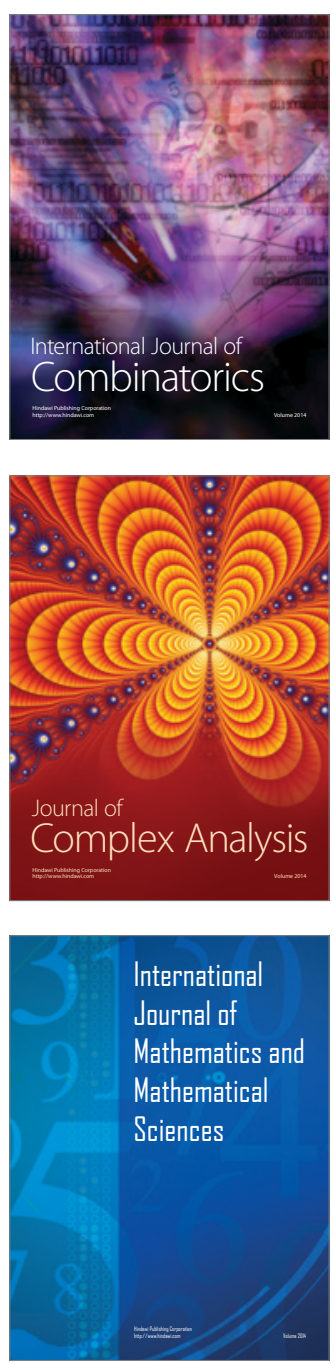
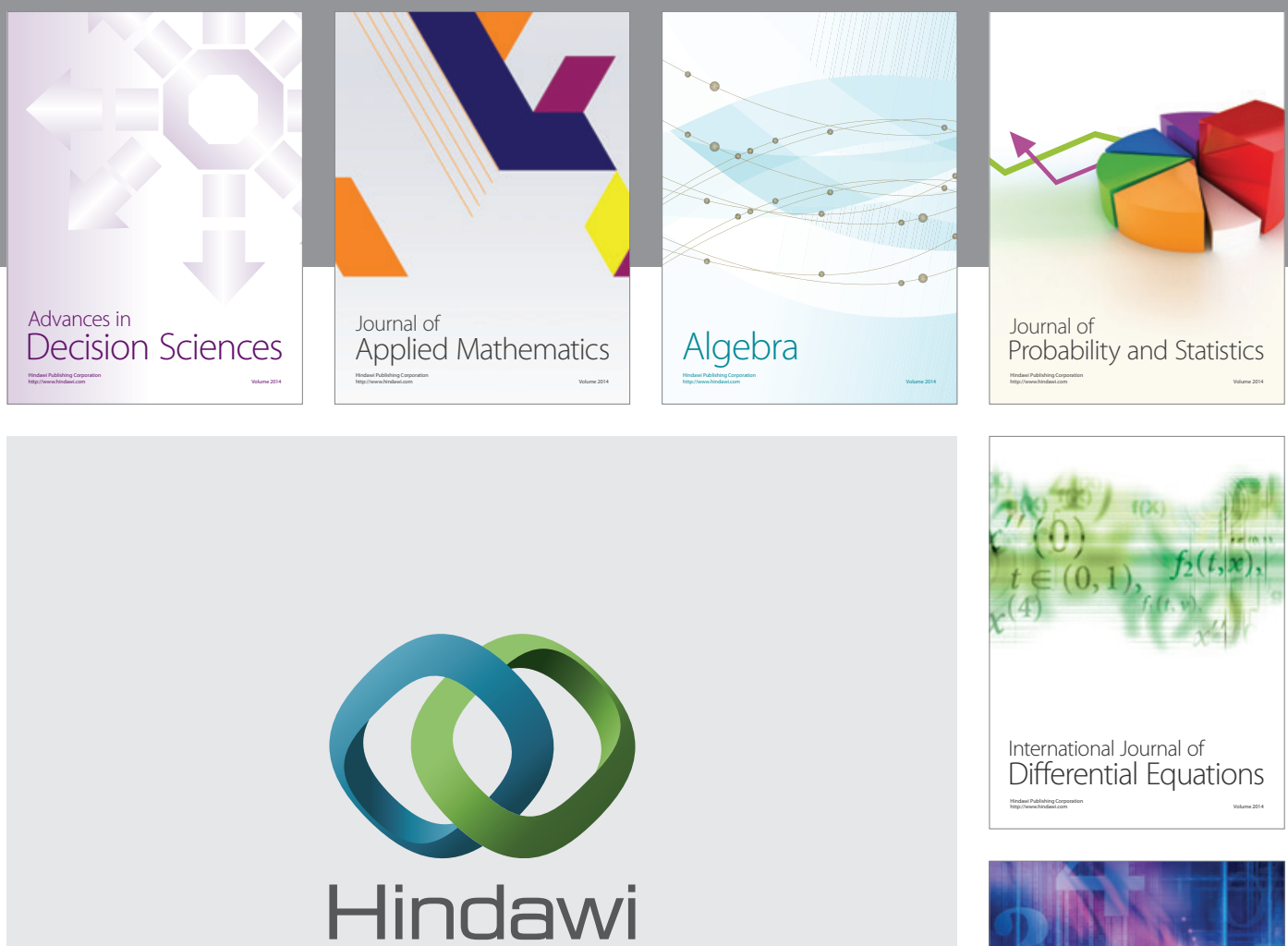

Submit your manuscripts at http://www.hindawi.com
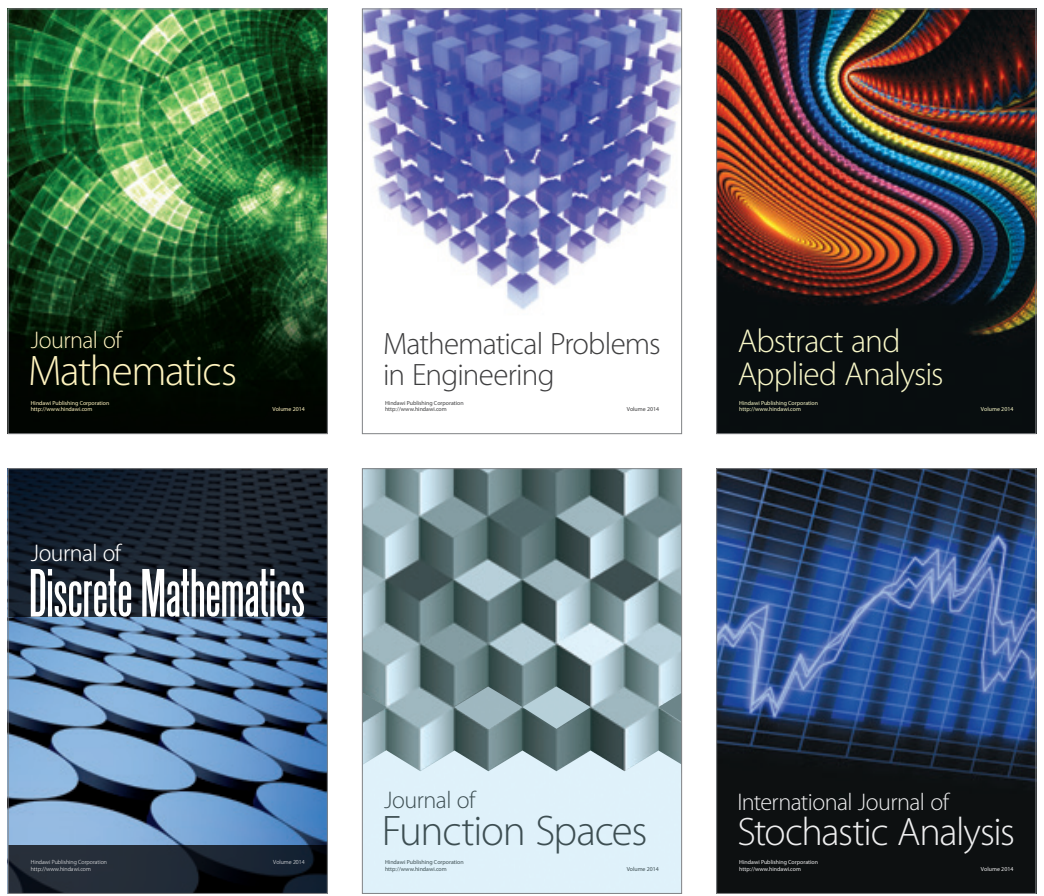

Journal of

Function Spaces

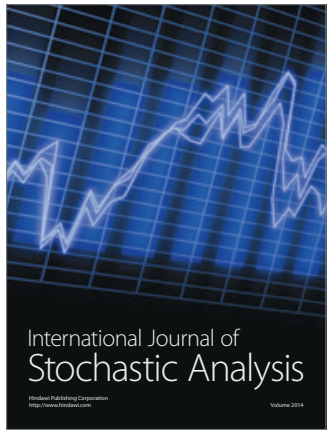

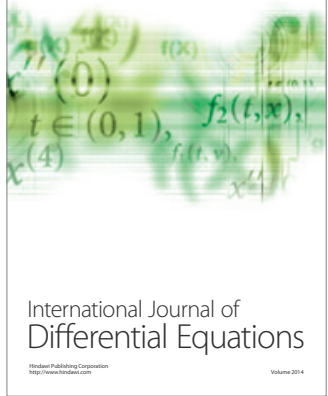
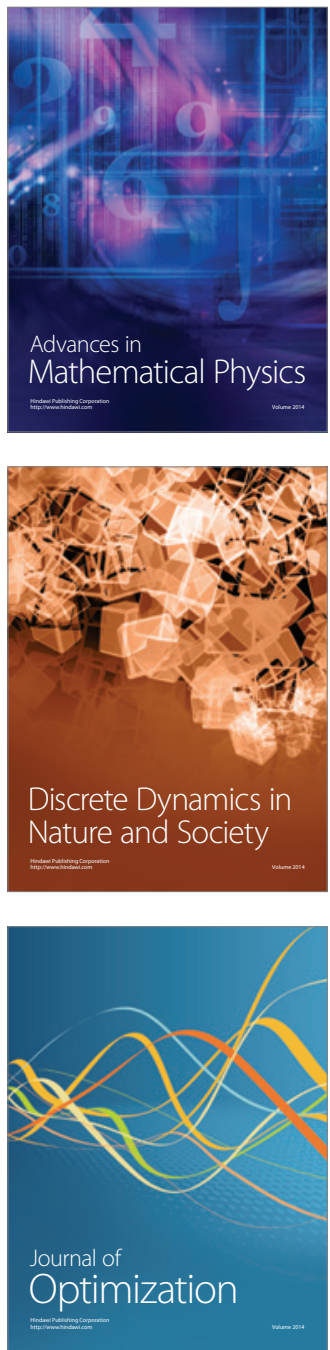